\title{
Path Analysis of Multitrait-Multimethod Matrices
}

\author{
Neal Schmitt \\ Michigan State University
}

\begin{abstract}
Path analyses of two multitrait-multimethod matrices are used as examples of the kind of information afforded by application of the technique. Discussed are tests of various possible models of the matrix, the reproduction of several correlations, and various reduced models. It is concluded that the technique should be of considerable aid to researchers who want to evaluate the convergent and discriminant validity of their measures.
\end{abstract}

A fundamental problem in the validation of both psychological tests and hypothetical constructs in the behavioral sciences is the assessment of their convergent and discriminant validity. Campbell and Fiske (1959) pointed out that in order to demonstrate construct validity, an investigator must show that a test correlates highly with the other variables with which it should theoretically correlate and that it does not correlate significantly with the variables from which it should differ. The former set of correlations indicates convergent validity; the latter, discriminant validity.

In order to examine more systematically the convergent and discriminant validity of a set of measures, Campbell and Fiske (1959) proposed the use of a multitrait-multimethod matrix (MTMM) of correlations. This procedure re-

APPLIED PSYCHOLOGICAL MEASUREMENT

Vol. 2. No. 2 Spring 1978 pp. 157-173

(C) Copyright 1978 West Publishing Co. quires (1) the assessment of two or more traits by two or more methods and (2) the computation of all possible correlations among scores on the traits measured by different methods. An hypothetical MTMM matrix is illustrated in Table 1. The diagonal submatrices are referred to as heterotrait-monomethod blocks and represent the correlations among Traits A, B, and C measured by a single method. The off-diagonal submatrices, called heterotrait-heteromethod blocks, are the correlations among traits measured by different methods. The diagonal values in these blocks (labeled as $V$ in Table 1) are the correlations between different measures of the same trait and are termed validities.

Four criteria have been suggested by Campbell and Fiske for evaluating the MTMM matrix. First, the correlations between similar traits measured by different methods (i.e., the convergent validities) should be both statistically significant and high enough to warrant further consideration. Second, the convergent validities should be higher than the correlations between different traits measured by different methods; i.e., the off-diagonal elements in the heteromethod submatrices. Third, the convergent validities should be higher than the correlations between different traits measured by the same method, i.e., the off-diagonal elements in the monomethod submatrices. Finally, a similar pattern of trait intercorrelations should be ap- 
Table 1

Multitrait-Multimethod Matrix ${ }^{a}$

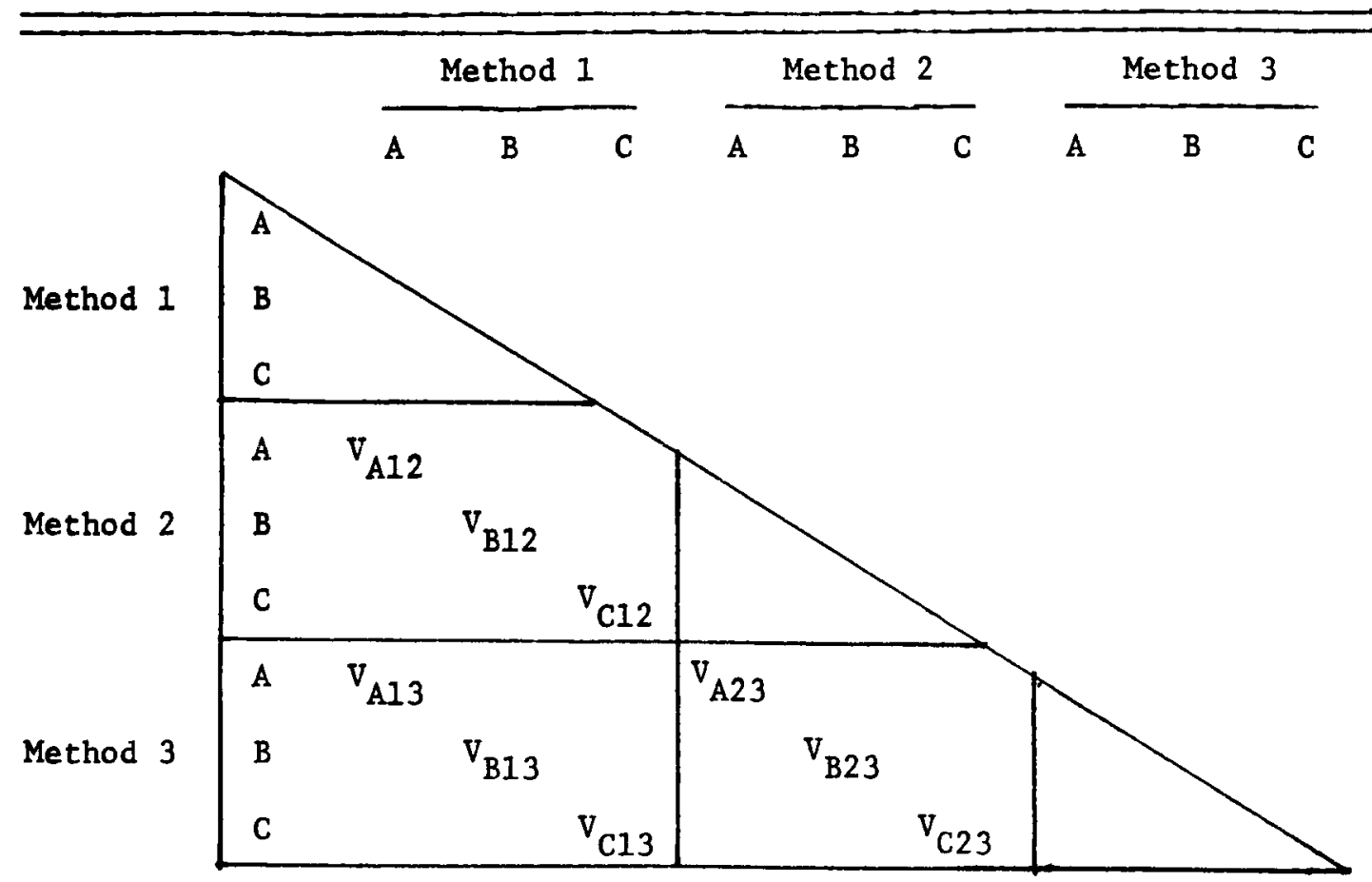

\section{${ }^{a} A, B$, and $C$ represent different traits, $V_{A 12}, V_{B 12}$, etc. are the validities or the correlations between different measures of the same trait.}

parent in the heterotrait-monomethod submatrices and the heterotrait-heteromethod submatrices.

Analysis of construct validity using the MTMM criteria has been frequent in the behavioral sciences. For example, ratings of the same traits by peers, subordinates, and supervisors have been compared by Lawler (1967); and ratings of blacks and whites on the same traits have been compared by Schmidt and Johnson (1973). Campbell and Fiske's original example (1959) involved the examination of measures of dominance, sociability, and achievement motivation by self-report inventory, projective technique, and associates' ratings. Ostrom (1969) and
Kothandapani (1971) have utilized the MTMM matrix to compare the ability of Likert, Guttman, and Thurstone scaling procedures to measure three hypothesized components of attitudes. Sociologists have frequently compared interview and questionnaire measures of the same constructs (Althauser, Heberlein, \& Scott, 1971). Although the methodology has been adapted to clarify a wide variety of problems, ambiguities with respect to the criteria remain.

The purpose of the present paper is to examine a path analytic strategy of analyzing the MTMM matrix. The general outline for the use of path analysis to examine the MTMM matrix has been presented by Werts and Linn 
(1970). An example of the Werts and Linn proposal has been given by Kalleberg and Kluegel (1975), and Kenny (1976) has suggested tests of hypotheses concerning various elements of the path analytic formulation. However, a single full presentation of the purposes which the path analysis approach to MTMM matrices can serve is necessary to facilitate its use by a greater number of researchers interested in the construct validity of their instruments. The objective of the present paper is to provide that presentation.

Several problems have been noted with respect to the Campbell-Fiske criteria. First, and perhaps most obvious, is the subjectivity of the criteria employed; all that is possible is a greater than or lesser than comparison of correlations in various portions of the matrix. Second, this subjective criterion becomes almost impossible when more than a relatively few traits or methods are involved. Somewhat more subtle are problems which occur when the variables are measured with different reliabilities. What may appear to be discriminant or convergent validity for some traits and not others may simply be due to differential reliabilities. Finally, the implicit assumption in the Campibell-Fiske formulation that traits and methods are uncorrelated is probably not tenable in most situations; furthermore, it is difficult, if not impossible, to evaluate this assumption by visual examination of the MTMM matrix (Alwin, 1974; Kalleberg \& Kluegel, 1975). The confirmatory factor analysis described and illustrated in the present paper allows a more complete analysis of the extent to which each variable is affected by trait and method variance.

\section{Method}

Confirmatory factor analysis or path analysis allows investigation or evaluation of the extent to which measured variables can be explained on the basis of hypothesized underlying traits and methods. It is a method for describing the correlations among a set of standardized variables by use of an hypothesized model which represents the relationships among these variables. Figure 1 is an illustration of the path model generally used to represent the MTMM matrix (it has been represented by slightly different models, e.g., Kenny, 1976; Werts, Jöreskog, \& Linn, 1976). The path diagram implies that each observed variable contains variance attributable to traits, methods, and uniqueness.

This model implies nine structural equations-one for each observed variable in the form of Equation 1:

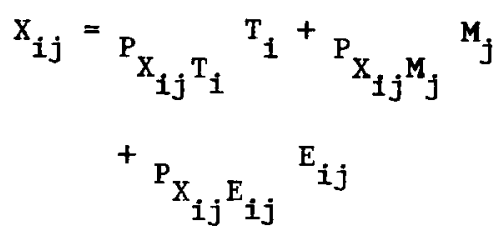

where $x_{i j}$ indicates an observed variable, corresponding to the $i^{\text {th }}$ trait, $T_{\text {, }}$, measured by the $j^{\text {th }}$ method of measurement, $M_{j}$; and $E_{\imath}$ is the uniqueness associated with a measured variable. The path coefficients are designated by $P$. In addition, there is allowance for the estimation of correlations among traits, among methods, and among traits and methods (indicated by the curved double headed arrows in Figure 1). To reproduce any correlation in the path diagram, the product of paths which lead to both observed variables is computed.

The correlation of $X_{1}$ and $X_{5}$, a heterotraitheteromethod correlation, is diagrammed in Figure 2. There are four sources of intercorrelation between these two measures. First, that due to traits is a product of the path from $T_{2}$ to $X_{5}$, the path from $T_{1}$ to $X_{1}$, and the intercorrelation between $T_{1}$ and $T_{2}$. Second, the intercorrelation due to commonality of methods is the product of the path from $M_{2}$ to $X_{5}$, the path from $M_{1}$ to $X_{1}$, and the intercorrelation between $M_{1}$ and $M_{2}$. Thirdly, the intercorrelation between $X_{1}$ and $X_{5}$ due to the intercorrelation of $T_{1}$ and $M_{2}$ is the product of the path from $M_{2}$ to $X_{5}$, the path from $T_{1}$ to $X_{1}$, and the intercorrelation between $T_{1}$ and $M_{2}$. Similarly, the intercorrelation be- 


\section{Figure 1}

Path diagram for a multitrait-multimethod matrix which includes three methods and three traits.

$X_{1}$ through $X_{9}$ represent 9 measured variables.

$T_{1}$ through $T_{3}$ represent three underlying traits, while $M_{1}$ through $M_{3}$ represent the three methods.

In addition to the curved lines indicating correlations among traits and among methods, there should be curved lines between each method and trait indicating the possibility of

trait-method correlations. $E_{1}$ through $E_{9}$ are the uniquenesses associated with each observed variable.

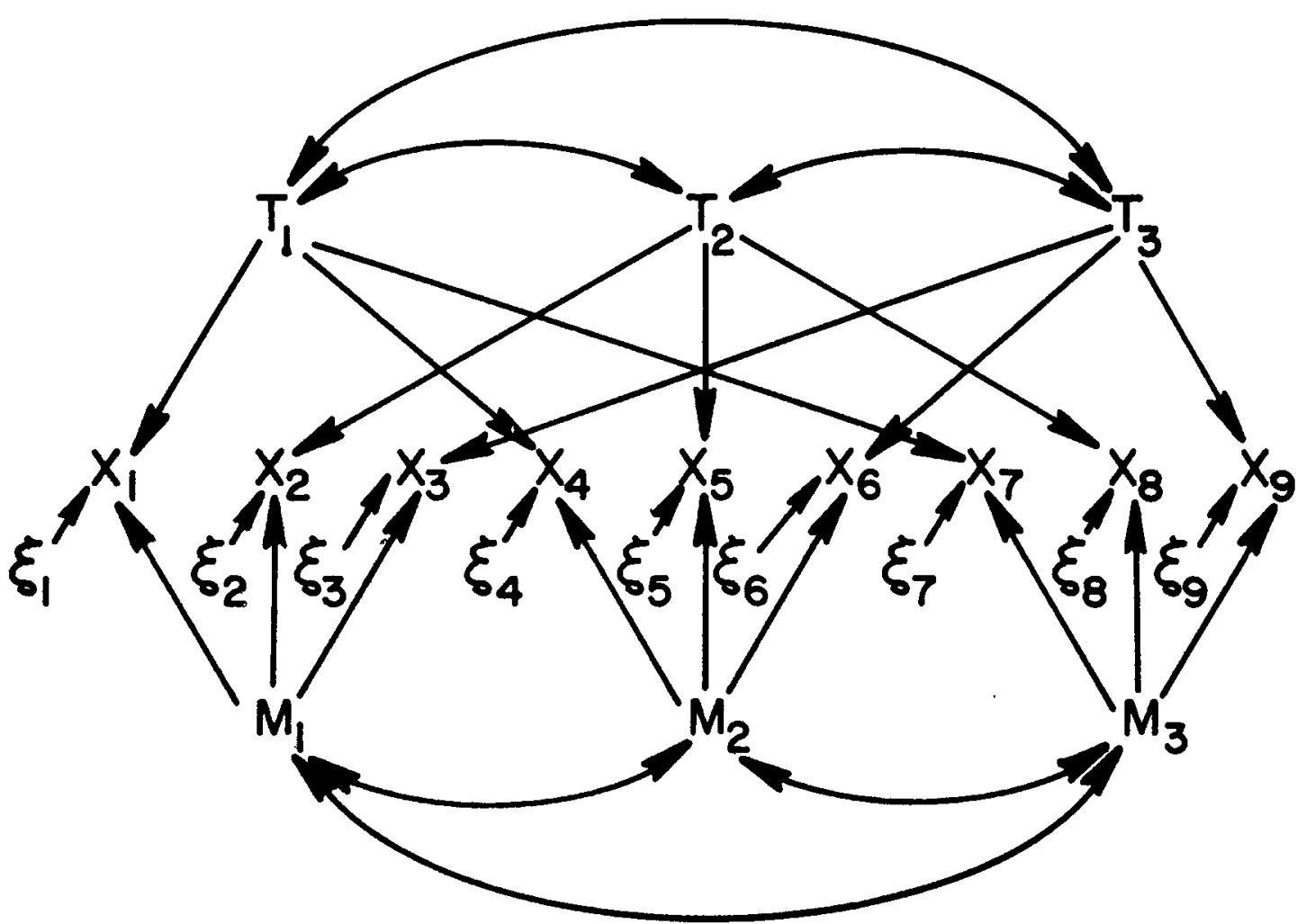

tween $X_{1}$ and $X_{5}$ due to the intercorrelation of $T_{2}$ and $M_{1}$ is the product of the path from $T_{2}$ to $X_{5}$, the path from $M_{1}$ to $X_{1}$, and the intercorrelation of $M_{1}$ and $T_{2}$.

Werts and Linn (1970) and Althauser and Heberlein (1970) have noted that estimation of all parameters suggested in Figure 1 requires at least three methods and three traits. In a threetrait $(p)$, three-method $(q)$ model, there are $[(p q)$ $(p q+1)] / 2$ or 45 observed correlations. The model in Figure 1 requires the estimation of 9 trait paths, 9 metnod paths, 9 uniquenesses, and 15 trait-method intercorrelations for a total of 42 parameters. With 45 observed correlations and 42 parameters to estimate, there are 3 degrees of freedom with which to test the model. Any fewer traits or methods will result in more parameters than observed correlations, hence an 


\section{Figure 2}

Example of paths between two different traits measured by two different methods.

Curved lines indicate intercorrelation

between underlying traits $(T$,$) and$ methods $\left(M_{\imath}\right)$. The $X_{t}$ represent observed variables.

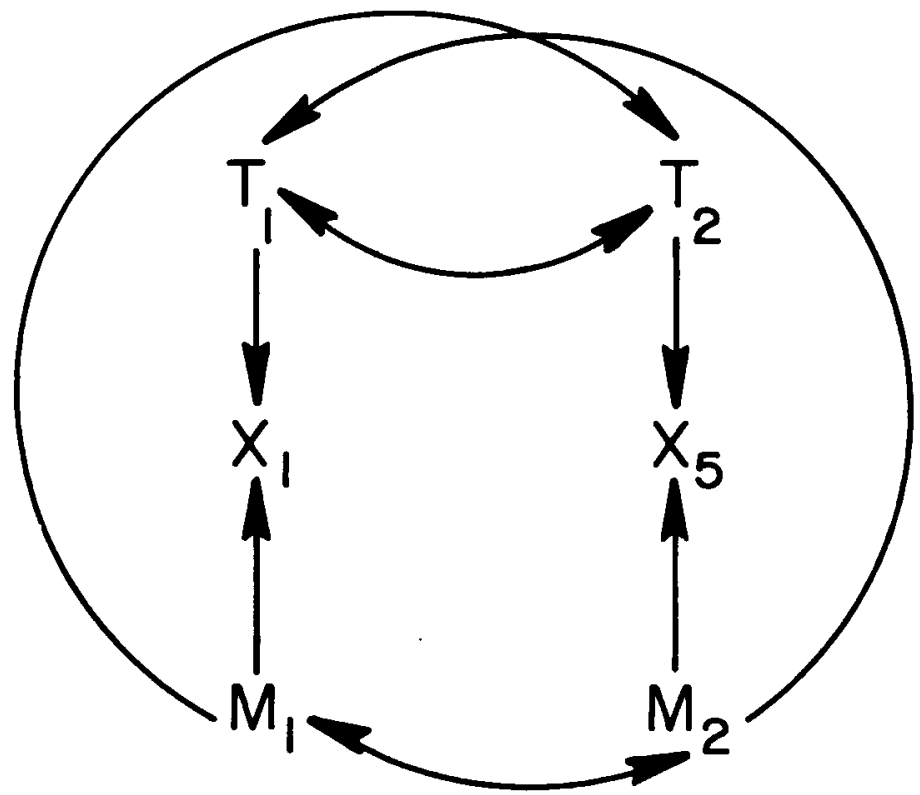

underidentified model. While this does present a problem in many applications of path analysis to MTMM matrices, some reduced models may be estimated and tested. These reduced models and the assumptions involved are discussed below.

In addition to specification of the sources of variance contributing to intercorrelations in the MTMM matrix, the degree of fit of any hypothesized model to the observed data can be tested. A $\chi^{2}$ test of fit is described by Jöreskog (1969, $1970,1973)$ and is part of the computer output of the LISREL program (Jöreskog \& VanThillo, 1972). The maximum likelihood method employed by this program minimizes the function ML as

$M L=\ln |\Sigma|+\operatorname{trace}\left(S \Sigma^{-1}\right)-\ln |S|-r$

where In refers to the natural logarithm;

$\Sigma$ is the maximum likelihood estimate of the variance-covariance matrix;
$S$ is the sample variance-covariance matrix; and $r$ is the number of observed variables.

The $\chi^{2}$ test used to evaluate the adequacy of the model is equal to $N(\mathrm{ML})$, where $N$ is the sample size. The degrees of freedom for this $\chi^{2}$ are equal to the number of elements in the sample variance-covariance matrix minus the number of independent parameters estimated. Comparisons of the degree of fit of alternative models can be made by taking the difference of the $\chi^{2}$ values for the two models. This difference is also distributed as a $\chi^{2}$, with degrees of freedom equal to the difference in degrees of freedom for the individual models. For example, discriminant validity implies absence of correlation among traits (or at least "low" intertrait correlations) and traits and methods. Computation of the $\chi^{2}$ value comparing the observed and reproduced correlations for the model in Figure 1 and the $\chi^{2}$ for a model in which correlations among traits 
and traits and methods are fixed at zero, provides the basis for a test of the significance of these correlations. This is true only in the comparison of "nested models," that is, models where no new variables are specified and constraints on the relationships among the specified variables are imposed or relaxed (Jöreskog \& Goldberger, 1975; Kenny, 1976).

Since the $\chi^{2}$ test is dependent on sample size, a much better index of the fit of any given model is the residual correlation matrix-the difference between the reproduced and observed correlation matrix. As for other tests of significance, with large sample size the observed corre- lation matrix will almost always be significantly different from the reproduced correlation matrix. With small sample size, nearly any model will provide adequate fit. Ultimately, the addition of parameters should be decided on conceptual and theoretical grounds; but a $\chi^{2} / d f$ ratio larger than 10 indicates an inadequate fit, while a $\chi^{2} / d f$ ratio less than 1.00 indicates the model fits too well. Such a model would be unlikely to remain stable in future samples.

As a final degree of fit measure, Tucker and Lewis (1973) have proposed a reliability coefficient which is a ratio of the amount of covariation explained by a proposed model to the total

Table 2

Multitralt Multimethod Data Sets $^{a}$

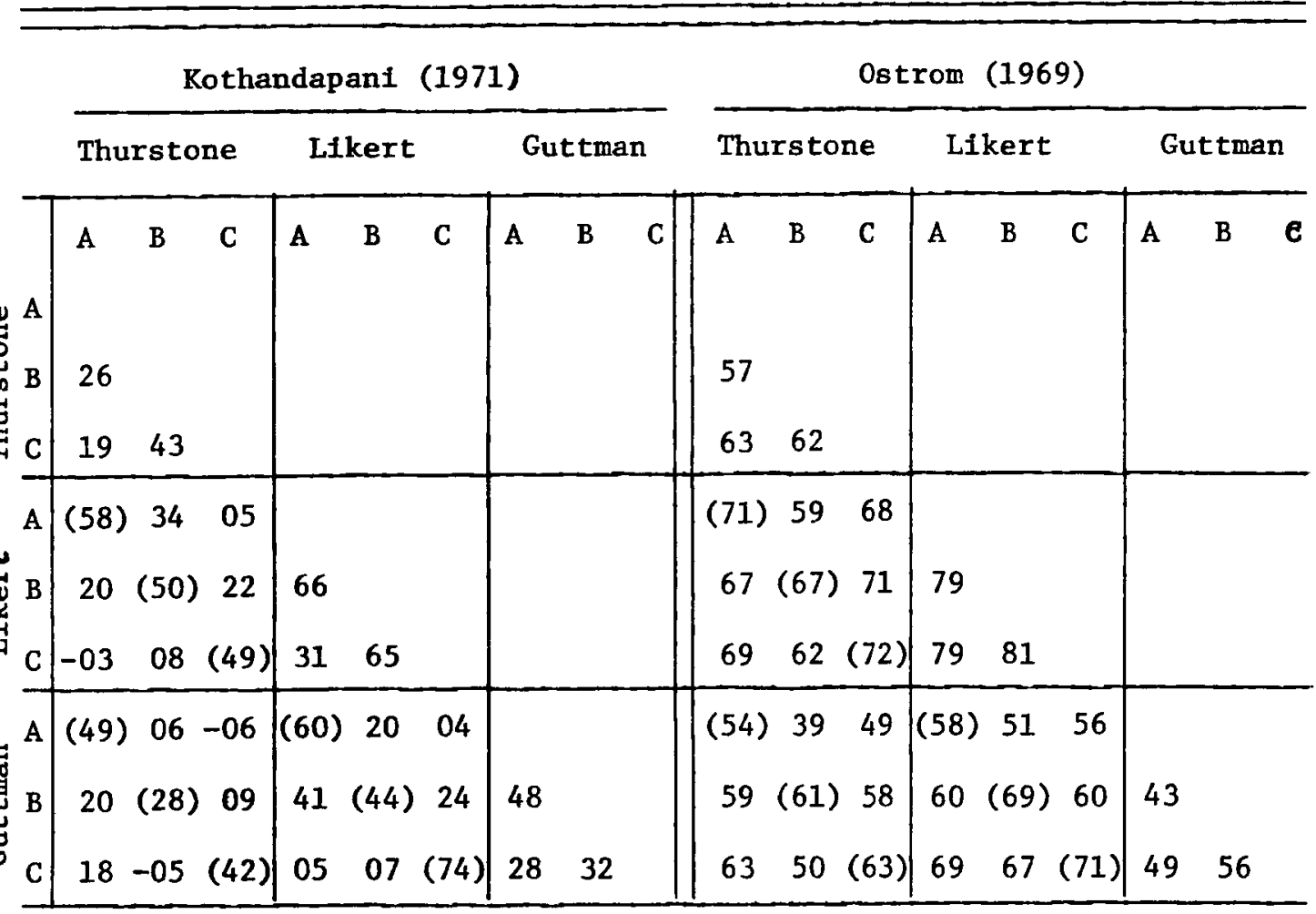

${ }_{A} A, B, C$ refer to affective, behavior, and cognitive attitudinal components for the Ostrom data set and feeling, belief, and Intention to act components for the Kothandapant data. Figures in parentheses are the validities. 
Table 3

Kothandapan1 Matrix - Estimated Path Coefficients

for the Model in Figure 1

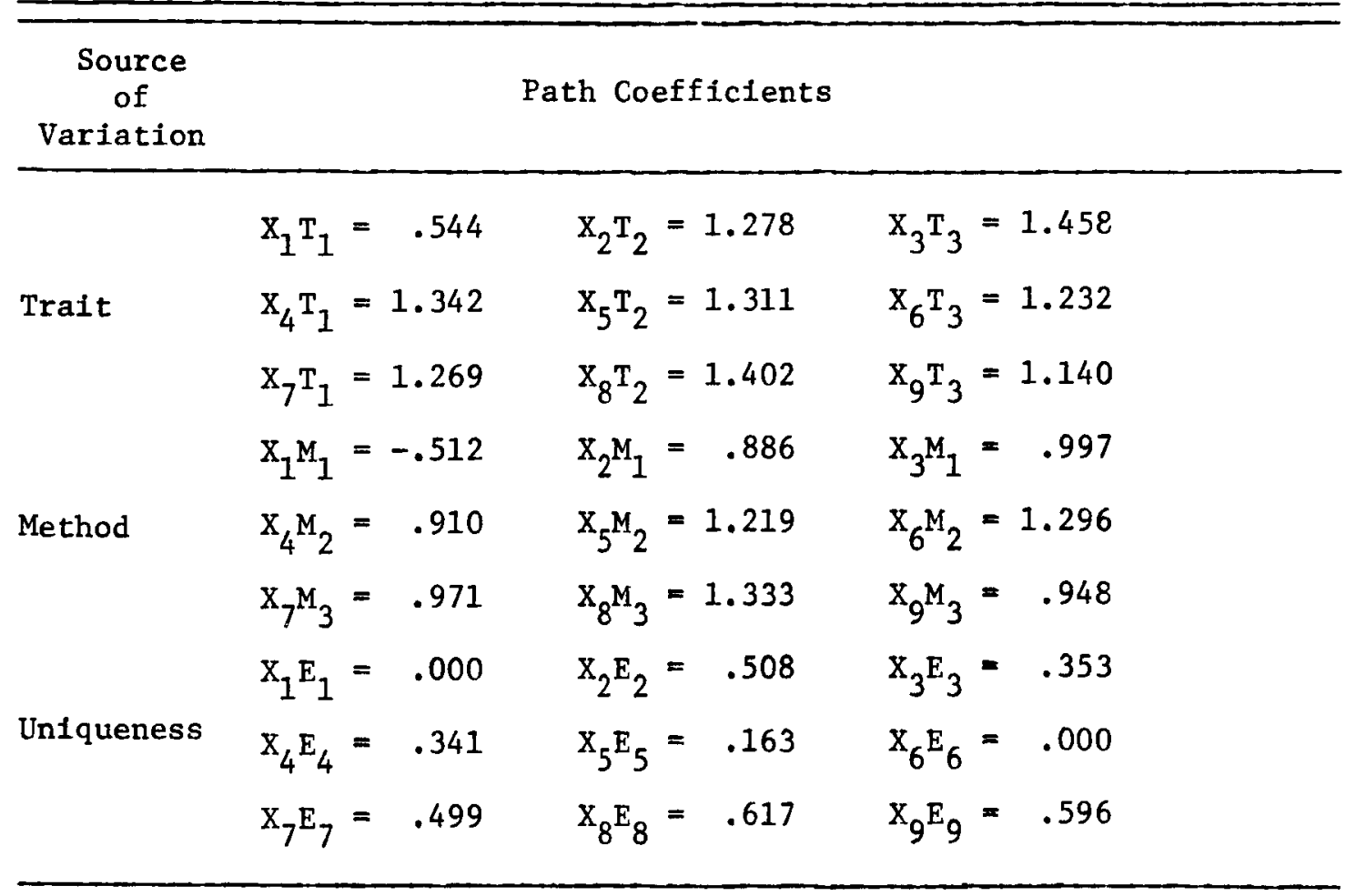

amount of covariation available to be explained by a model. Computationally, this formula takes the following form:

$\rho=\frac{C_{0}-C_{q}}{C_{0}-E\left(C_{q}\right)}$

where $C_{o}$ equals the sum of the squares of the off-diagonal elements of the sample variancecovariance matrix divided by $[r(r+1) / 2]$;

$C_{q}$ equals $\chi_{2} / N$ divided by the degrees of free-

dom associated with the $\chi^{2}$; and

$E\left(C_{q}\right)=1 / N$.

They suggest that values greater than .90 represent quite adequate fit.

To illustrate the utility of confirmatory factor analysis in the examination of MTMM matrices, two sets of data will be used. Both involve measures of affective, behavioral, and cognitive as- pects of attitude using Likert, Thurstone, and Guttman scaling procedures. The first set of data has been presented by Kothandapani (1971) and represents a matrix which by visual inspection meets the Campbell-Fiske criteria. The second data set comes from a study by Ostrom (1969) in which the extent to which the Campbell-Fiske criteria are met is not as clear. For both of these data sets, the observed MTMM matrix is presented along with the path analytic solution as diagrammed in Figure 1, some alternate models of the observed relationships, and the degree of fit represented by these models. In addition, several examples of reproduced correlations are presented as illustrations of the detail a path analytic approach provides concerning the implications of an hypothesized model. 
Table 4

Ostrom Matrix - Estimated Path Coefficients

for the Model in Figure 1

Source

of

Variation
Path Coefficients

\begin{tabular}{|c|c|c|c|}
\hline & $\mathrm{X}_{1} \mathrm{~T}_{1}=1.072$ & $\mathrm{x}_{2} \mathrm{~T}_{2}=1.142$ & $\mathrm{x}_{3} \mathrm{~T}_{3}=1.058$ \\
\hline \multirow[t]{3}{*}{ Trait } & $\mathrm{x}_{4} \mathrm{~T}_{1}=.609$ & $\mathrm{x}_{5} \mathrm{~T}_{2}=-.021$ & $x_{6} T_{6}=$ \\
\hline & $x_{7} T_{1}=.640$ & $\mathrm{X}_{8} \mathrm{Y}_{2}=.555$ & $\mathrm{X}_{9} \mathrm{~T}_{9}=.734$ \\
\hline & $\mathrm{X}_{1} \mathrm{M}_{1}=.371$ & $\mathrm{X}_{2} \mathrm{M}_{1}=.416$ & $\mathrm{x}_{3} \mathrm{M}_{1}=.388$ \\
\hline \multirow[t]{3}{*}{ Method } & $x_{4} M_{2}=.341$ & $\mathrm{X}_{5} \mathrm{M}_{2}=1.015$ & $\mathrm{X}_{6} \mathrm{M}_{2}=$ \\
\hline & $\mathrm{X}_{7} \mathrm{M}_{3}=$ & $\mathrm{x}_{8} \mathrm{M}_{3}=.597$ & $\mathrm{X}_{9} \mathrm{M}_{3}=$ \\
\hline & $x_{1} E_{1}=$ & $X_{2} E_{2}=.003$ & $X_{3} E_{3}=.569$ \\
\hline \multirow[t]{2}{*}{ Uniqueness } & $X_{4} E_{4}=.453$ & $X_{5} E_{5}=-.001$ & $X_{6} E_{6}=.424$ \\
\hline & $x_{7} E_{7}=$ & $\mathrm{X}_{8} E_{8}=$ & $X_{9} E_{9}=$ \\
\hline
\end{tabular}

\section{Results}

The Kothandapani (1971) and Ostrom (1969) data are presented in Table 2 . These matrices appear to be quite different when evaluated against the Campbell-Fiske criteria. The Ostrom matrix is characterized by very high intercorrelations among traits and validities which evidence very little difference from the remaining values in their respective rows and columns. The Kothandapani matrix, on the other hand, has lower validities; but, for the most part, these validities are markedly higher than values in their respective rows and columns. The Kothandapani data also exhibit relatively higher correlations between the affective and behavioral components of attitude than between other components for all monomethod and heteromethod blocks. By the Campbell-Fiske criteria, then, it could be concluded that the Kothandapani data represent a higher degree of convergent and discriminant validity than do the Ostrom data.

The LISREL program (Jöreskog \& VanThillo, 1972) described above was used to estimate the standardized path coefficients in Figure 1 for both data matrices presented in Table 2 . These coefficients for the Kothandapani matrix and the Ostrom matrix are presented in Tables 3 and Table 4, respectively. In addition, the intercorrelations among traits, among methods, and among traits and methods are presented in Table 5. Table 3 indicates that considerable trait 
Table 5

Intercorrelation of Trait and Method Factors

\begin{tabular}{|c|c|c|c|c|c|c|c|c|c|c|}
\hline & \multicolumn{5}{|c|}{ Kothandapani Matrix } & \multicolumn{5}{|c|}{ Ostrom Matrix } \\
\hline & $\mathrm{T}_{1}$ & $\mathrm{~T}_{2}$ & $\mathrm{~T}_{3}$ & $\mathrm{M}_{1}$ & $\mathrm{M}_{2}$ & $\mathrm{~T}_{1}$ & $\mathrm{~T}_{2}$ & $\mathrm{~T}_{3}$ & $\mathrm{M}_{1}$ & $\mathrm{M}_{2}$ \\
\hline $\mathrm{T}_{2}$ & .88 & & & & & .79 & & & & \\
\hline $\mathrm{T}_{3}$ & .70 & .80 & & & & .95 & .83 & & & \\
\hline $\mathrm{M}_{1}$ & -.79 & -.74 & -.77 & & & -.75 & -.50 & -.72 & & \\
\hline $\mathrm{M}_{2}$ & -.72 & -.70 & -.69 & .73 & & .74 & .72 & .79 & -.33 & \\
\hline$M_{3}$ & -.73 & -.84 & -.72 & .68 & .77 & .28 & .06 & .24 & .22 & .50 \\
\hline
\end{tabular}

variance exists in the Kothandapani matrix, with the exception perhaps of the $X_{1}$ variable, but method and unique variance also account for some of the variance in the Kothandapani measures. Table 5 indicates that there are high correlations among traits and methods and high negative correlations between methods and traits. The trait and trait-method intercorrelations may be considered as indications of a lack of discriminant validity. However, there are instances when some trait intercorrelation would be consistent with the substantive nature of the traits. This would certainly be true of the data sets used in this paper.

The path coefficients for the Ostrom matrix presented in Table 4 indicate that the Thurstone scales (Method 1) are most associated with trait variance and that Trait 2 (the behavioral component) loads heavily on the second method (Likert scale) factor. In addition, Table 5 indicates substantial correlation between the affective and cognitive components $(r=.95)$ and relatively high trait-method intercorrelations. Finally, it appears that the Thurstone methodology yields different measures of the three attitudinal components as evidenced by the negative correlations between Method 1 and each of the traits, and the correlations between Method 1 and the other two methods.

Examination of these path coefficients can identify problems in the measurement of particular traits or problems with a given method. The extent to which these problems represent significant departures from convergent or discriminant validity can be evaluated by the $\chi^{2}$ test mentioned previously. In turning to the evaluation of various models of both correlation matrices, it should be pointed out that the $\chi^{2}$ test is dependent on sample size and that it may also be desirable to pay attention to relative differences in $\chi^{2}$ from different models. With very large sample sizes, it may be desirable to use the $\chi^{2}$ :degree of freedom ratio, the average of the residual correlation matrix as an index of the degree of fit, or the Tucker and Lewis (1973) reliability estimate.

\section{Evaluation of Alternate Models of the Multitrait-Multimethod Matrices.}

The path model described in Figure 1 was altered successively in two ways. First, different portions of the correlations in Table 5 were fixed at zero. Secondly, different numbers of trait and 


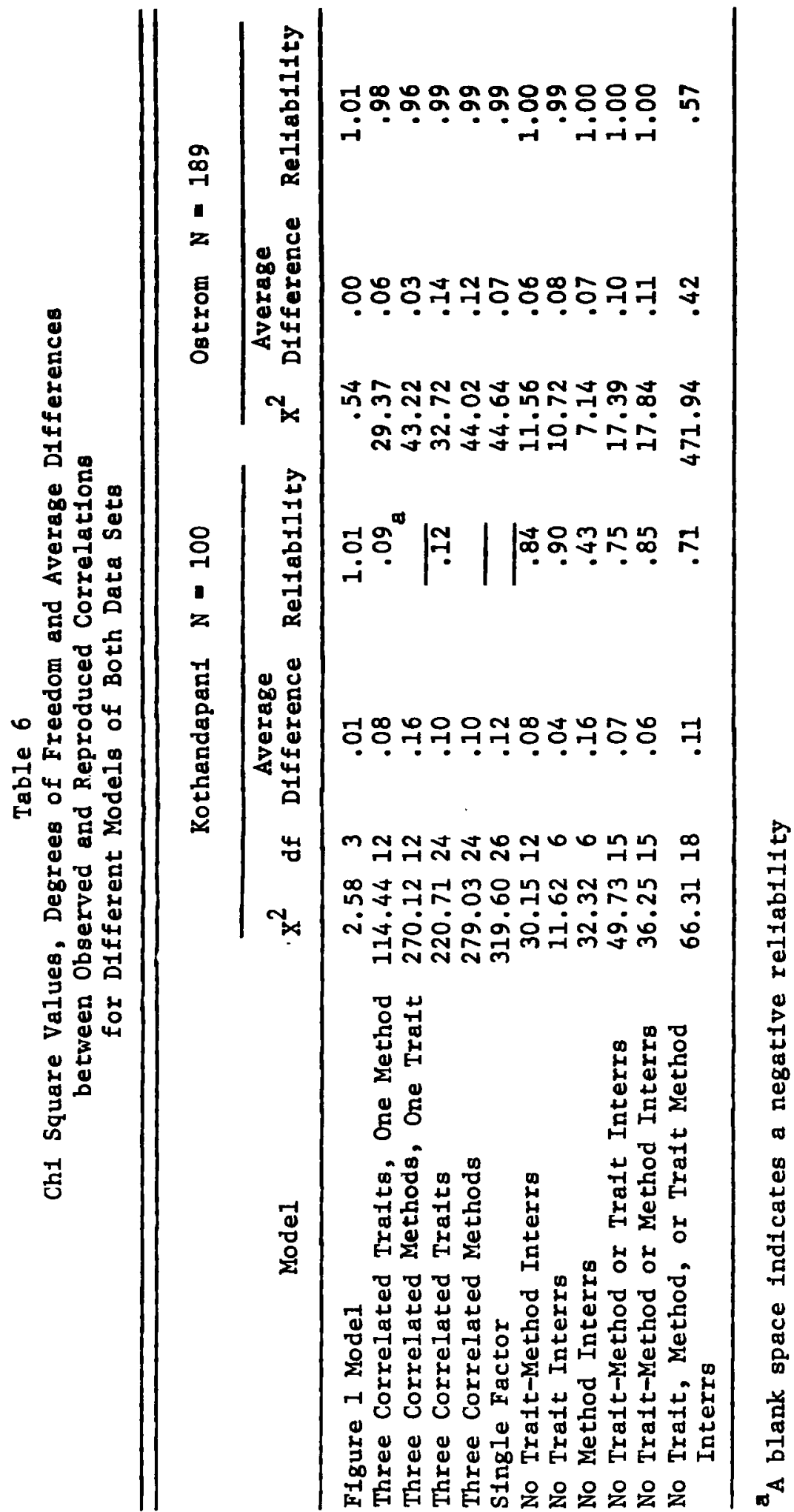

Downloaded from the Digital Conservancy at the University of Minnesota, http://purl.umn.edu/93227. 
method factors were specified, beginning with an evaluation of the extent to which a single factor accounted for the total matrix and ending with the full model represented by Figure 1. For the various models evaluated, their associated degrees of freedom and chi-squares and the average absolute difference between observed and reproduced correlations are presented in Table 6 for both the Ostrom and Kothandapani data. Loadings on trait or method factors could also be constrained to be equal to assess the degree to which each method measured similar portions of trait variance or the extent to which traits were similarly affected by method variance as Kenny (1976) has suggested.

A test of the extent to which each matrix meets the criteria for discriminant validity can be made by comparing the chi-square for the full model with the chi-square for the model in which the trait-method and trait intercorrelations are fixed at zero, as described in the introduction. For the Kothandapani matrix, the full model yielded a chi-square of 2.58 with three degrees of freedom. With the restrictions listed above, chi-square was 49.73 with 15 degrees of freedom. The difference in these chi-squares, 46.85 , is a chi-square variable with degrees of freedom equal to the difference in degrees of freedom of the two models-in this case, 12. Since $\chi_{12}^{2}=46.85$ is significant at the $p<.001$ level, the conclusion is that these criteria for discriminant validity were not completely met.

This use of chi-square is appropriate when comparing "nested" models, i.e., models where no new variables are specified but restrictions or constraints are placed on the variables already in the model. Whether or not it should be said that trait intercorrelations are zero would depend on the nature of the traits. For example, whether or not any discriminant validity was displayed could be tested by fixing the trait intercorrelations at 1.00 and comparing the resultant chi-square with the original full model chisquare. The level of discriminant validity of the Kothandapani matrix is evidenced by comparing the single factor model $\left(\chi_{26}^{2}=319.60\right)$ and the three-trait model $\left(\chi_{24}^{2}=220.71\right)$.
Since new variables have been specified, using the chi-squares as relative degrees of fit, the test described above comparing these two models is inappropriate. It is therefore clear that more than a single factor is involved. In addition, the average difference between the observed and reproduced correlation matrices indicated a better degree of fit for the three-trait model (see column headed "Average Difference" in Table 6). The Tucker and Lewis (1973) reliability coefficient led to similar conclusions; that is, there are distinct method and trait sources of variance. Models which do not include specification of three traits and three methods yielded near zero or negative reliabilities.

Similar examination of the Ostrom matrix did not yield the same conclusions. The single factor model came reasonably close to explaining the Ostrom matrix; and it appears, by comparing the other models presented, that differences in correlations in the Ostrom matrix were probably more the result of different methods than the result of different traits. The fit of a three-method, one-trait model was superior to that of a threetrait, one-method model. Any model which allowed for correlation among variables yielded a reasonably good fit to the data.

In making successive chi-square comparisons of "nested" models, it is, of course, true that the probability level of these tests is altered and that specification of models becomes speculative and exploratory. In addition, as noted previously, the significance of chi-square is directly dependent on sample size. Hence, the reliability index and differences between observed and actual correlation matrices are probably best employed as descriptive and relative indicators of the fit of various models of a MTMM matrix.

\section{Decomposition of Reproduced Correlations}

In addition to the overall indications of construct validity which are illustrated in the previous section, path analytic techniques allow the decomposition of individual correlations in the reproduced correlation matrix. This procedure 


\section{Table 7}

Examples of Decomposition of Reproduced CorrelationsKothandapani Data

Heterotrait-Monomethod $\left(r_{x \mid x 3}=.189\right)$

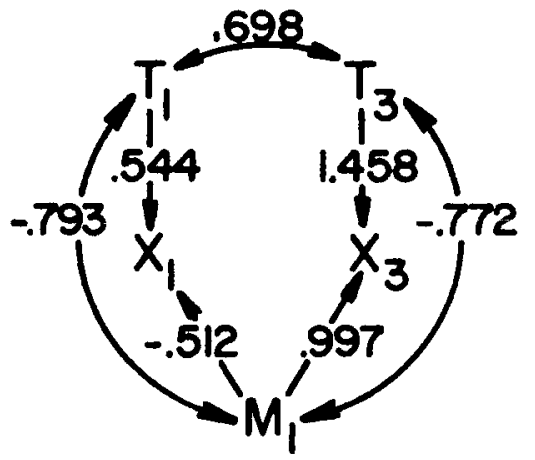

$(.544)(.698)(1.458)=.544-$ Trait

$(-.512)(.997) \quad=-.510-$ Method

$(1.458)(-.772)(-.512)=.576\}$ Trait - Method

$(.544)(-.793)(.997)=-.430\}$ Interoctions

Heterotrait-Heteromethod $\left(r_{X \mid \times 5}=.199\right)$

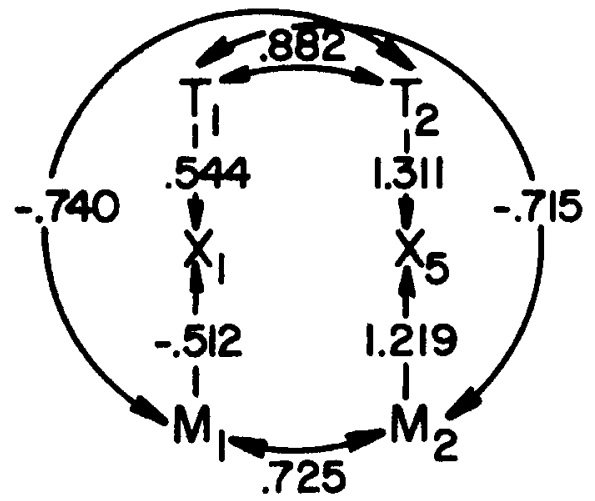

$(.544)(.882)(1.311)=.629-$ Trait

$(-.512)(.725)(1.219)=-.452-$ Method

$(-.512)(-.740)(1.311)=.497\}$ Trait-Method

$(.544)(-.715)(1.219)=-.474\}$ interactions

Monotrait-Heteromethod $\left(r_{X \mid X 4}=.583\right)$

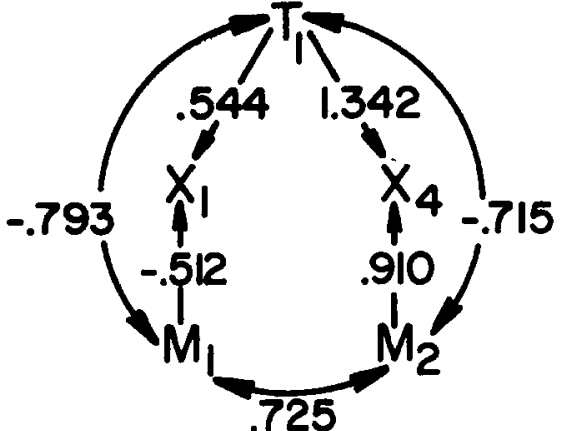

$(.544)(1.342)=.730-$ Trait

$(-.512)(.725)(.910)=-.388-$ Method

$(-.793)(-.512)(1.342)=.545\}$ Trait-Method

$(.910)(-.715)(.544)=\frac{-.354}{.583}$ Interactions

Downloaded from the Digital Conservancy at the University of Minnesota, http://purl.umn.edu/93227.

May be reproduced with no cost by students and faculty for academic use. Non-academic reproduction 


\section{Table 7 (continued)}

Examples of Decomposition of Reproduced Correlations Ostrom Data

Heterotrait-Monomethod $\left(r_{x \mid x 3}=.631\right)$

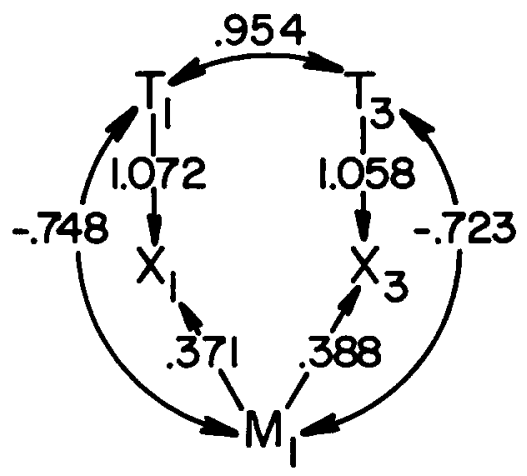

$(1.072)(1.058)(.954)=1.082-$ Trait

$(.371)(.388)=.144-$ Method

$(.371)(-.723)(1.058)=-.284)$ Trait-Method

$\left.(.388)(-.748)(1.072)=\frac{-.311}{.631}\right\}$ Interactions

Heterotrait-Heteromethod $\left(r_{x \mid \times 5}=.671\right)$

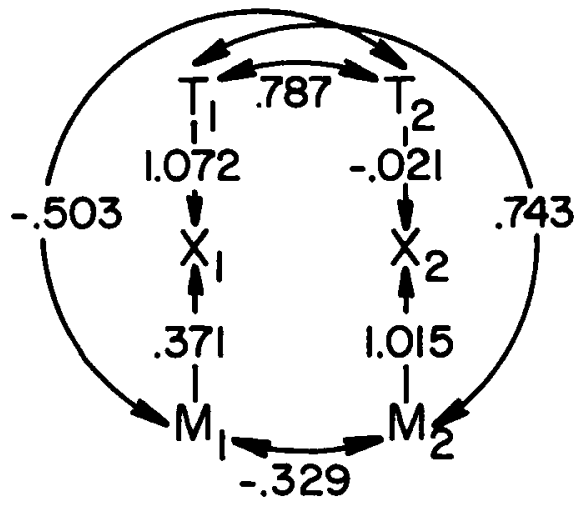

$(1.072)(.787)(-.021)=-.018-$ Trait

$(.371)(-.329)(1.015)=-.124-$ Method

$(1.015)(.743)(1.072)=.808\}$ Trait-Method

$\left.(.371)(-.503)(-.021)=\frac{.004}{.670}\right\}$ interactions

Monotrait-Heteromethod $\left(r_{X 1 \times 4}=.713\right)$

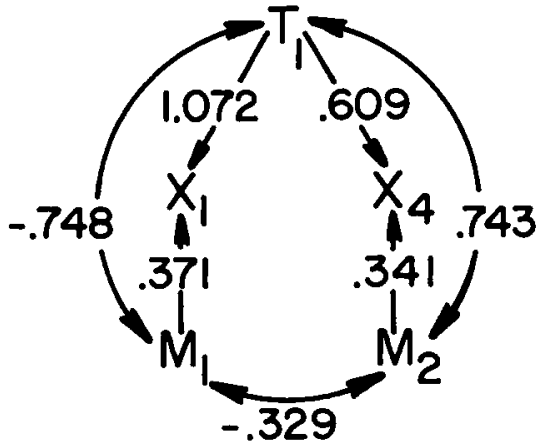

$(1.072)(.609)=.653-$ Trait

$(.371)(.341)(-.329)=-.042-$ Method

$(.341)(.743)(1.072)=.272\}$ Trait - Method

$\left.(.371)(-.748)(.609)=-\frac{.169}{.714}\right\}$ interactions

Downloaded from the Digital Conservancy at the University of Minnesota, http://purl.umn.edu/93227. 
was discussed in the introduction, and the decomposition of an heterotrait-heteromethod correlation was outlined in Figure 2. The decomposition of an heterotrait-monomethod correlation, an heterotrait-heteromethod correlation, and a monotrait-heteromethod correlation for both matrices is illustrated in Table 7 . In each case, the decompositions indicate why a pair of variables are correlated.

A particularly revealing decomposition is that of $r_{x \mid x s}$ for the Ostrom matrix. Almost all of the correlation between these two measured variables is due to the commonality between Method 2 and Trait 1 . There is little or no commonality due to traits or methods. For both data sets the trait contribution to the monotrait-heteromethod correlation is high, indicating substantial agreement between Likert and Thurstone scales in the measurement of the affective attitudinal component, which is a positive indication of convergent validity. In the case of heterotrait-monomethod correlations, however, this part of the decomposition should be low, unless there is no discriminant validity.

The heterotrait-monomethod example from the Kothandapani matrix indicates some correlation due to similar traits; the Ostrom example indicates a great deal of common trait variance. These estimates are purely descriptive, and what is good or bad will depend on the investigators' research and/or theoretical objectives. The information gained by such an analysis, nevertheless, does focus attention on problems concerning convergent and discriminant validity of individual measures.

\section{Alternate Models of the MTMM Matrix}

Table 6 contains chi-square values for five different reduced models of the MTMM matrix. These models not only provide useful information, as discussed above, but also would be especially helpful when estimation of the parameters in the full model, depicted in Figure 1, is not possible. As noted previously, this would occur when there are less than three methods and three traits or the equivalent number of intercorrelations. The five reduced models listed in Table 6 are illustrated in Figure 3. These and other reduced models (Althauser, Heberlein, \& Scott, 1971) are useful in evaluating the extent to which various assumptions concerning the data matrix are important; and even though the full model of Figure 1 cannot be evaluated, they provide some information concerning discriminant and convergent validity as well.

For example, Figure 3e postulates a single factor, be it trait or method variance, that accounts for the intercorrelations among the variables. The numbers in Table 6 indicate that the Ostrom data much more closely fit this hypothesis than do the Kothandapani data. While this is also observable from the correlation matrices in Table 1, a similar conclusion may not be as easily reached when the number of variables is greater and/or the matrices are more like each other.

Comparisons of the models involving three traits with those involving three methods also are revealing: Postulation of three methods in the Ostrom data results in a better fit than the postulation of three traits. What this suggests is that variations in the MTMM matrix for the Ostrom data set are more attributable to differences in methods of measurement than to differences in traits. By comparing the various models in Table 6 and Figure 3, some idea can also be obtained of the extent of the relative importance of trait variance, random error variance, and systematic error variance due to both a general method factor and specific method factors.

For example, in the Kothandapani data set. changing the model from a Figure 1 model to one corresponding to Figure $3 \mathrm{a}$ resulted in a much less adequate fit to the observed correlation matrix. A smaller drop in fit occurred in going from a three-trait, one-method model to a three-trait model, suggesting that the method variance present was specific to the scaling methodology employed. Moving from a threetrait model to a single factor model resulted in 


\section{Figure 3}

Some alternate models of the MTMM matrix.

In all models, uniquenesses are also considered a part of the variance of each observed variable as in Figure 1.

In all diagrams, $T$, represent underlying traits, $M$, represent methods, and $X$, represent observed variables.

3a. Thiree Traits, One Method: All Traits and Method are Correlated

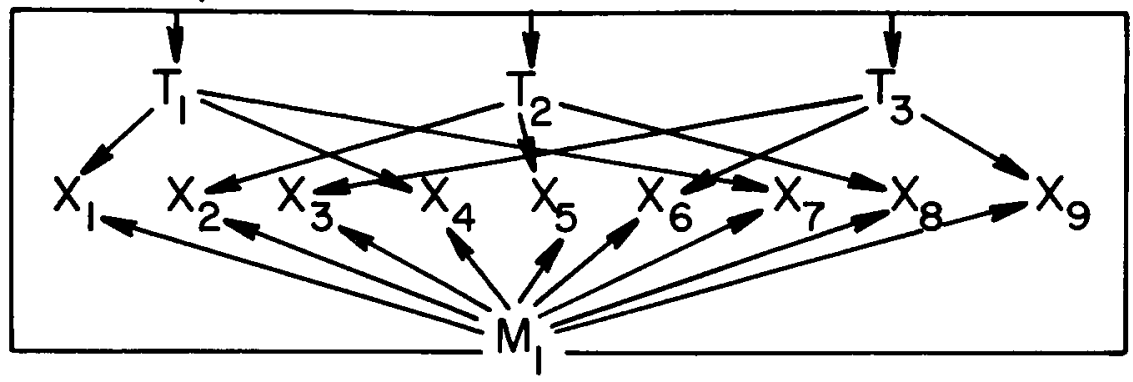

$3 \mathrm{~b}$. Three Methods, One Trait: All Methods and Trait are Correlated

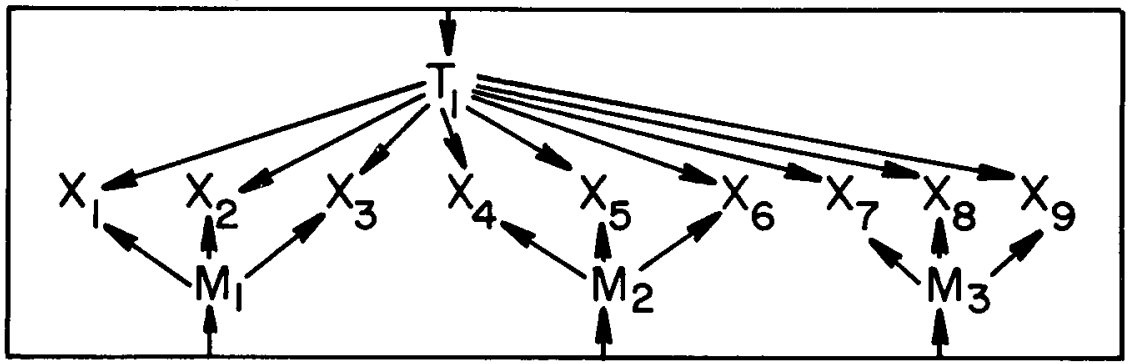

3 c. Three Traits: All Traits Correlated

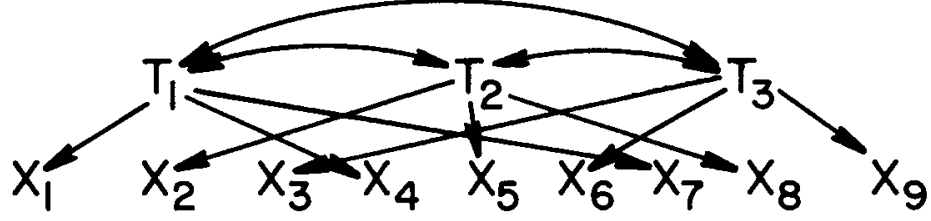

3 d. Three Methods: All Methods Correlated

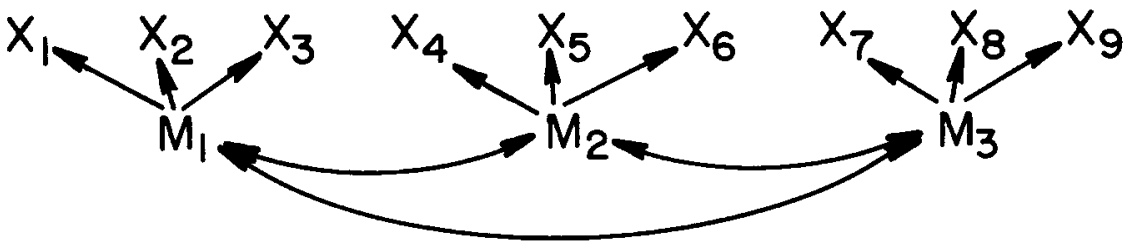

3 e. Single Factor

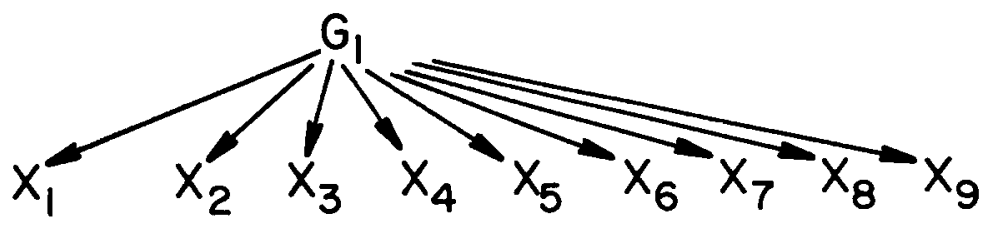

Downloaded from the Digital Conservancy at the University of Minnesota, http://purl.umn.edu/93227. 
additional relatively large drops in degree of fit to the observed correlation matrix, indicating that different traits were being measured. As noted above, similar comparisons for the Ostrom matrix actually indicate that the reverse is true; i.e., specification of independent methods resulted in better fit, while specification of independent traits resulted in a worse fit. It should also be pointed out that there are numerous other models which could be postulated. For example, models could be evaluated in which the loadings on traits measured by different methods or the loadings on methods factors are constrained to be equal.

\section{Conclusions}

The analyses presented in this paper should allow more detailed and intelligent analyses of MTMM data. Perhaps the most serious limitation of path analysis in this context is the inability to analyze in this manner MTMM matrices with less than three methods and three traits. However, with certain simplifying assumptions, such as those illustrated in the section on alternate models, components of smaller MTMM matrices can be evaluated. The strength of path analysis lies in the fact that it forces the researcher to make explicit his/her assumptions regarding the traits and methods employed. This should prove useful both to the researcher and the consumer of research.

Several other distinct advantages of the path analytic approach are important. First, decomposition of the reproduced correlation matrix allows the sources of variance which are contributing to correlations to be identified. Second, tests of specific hypotheses concerning portions of the model can be made. A word of caution concerning the use of these tests bears repetition. The test is dependent on sample size, which means that with small $N$, many different models will produce a correlation matrix nonsignificantly different from the observed correlation matrix. With large sample sizes, it is likely that no model will be nonsignificantly different from the observed correlation matrix. As suggested, the degree of fit of various models may be more appropriately indexed by the average difference between the observed and reproduced correlation matrix. Third, the extent to which different traits are a result of method and trait variance can be evaluated, and the various contributions to the correlations among traits and methods can be calculated. Because of the continued utilization of the MTMM matrix to explore a wide variety of questions and because the path analytic technique deals with the limitations of the Campbell-Fiske criteria noted in the introduction, this technique should be more frequently employed.

\section{References}

Althauser, R. B., \& Heberlein, T. A. Validity and the multitrait-multimethod matrix. In E. F. Borgatta. \& G. W. Bohrnstedt (Eds.), Sociological Methodology 1970. San Francisco: Jossey-Bass. 1970.

Althauser, R. P., Heberlein, T. A., \& Scott, R. A. A causal assessment of validity: The augmented multitrait-multimethod matrix. In H. M. Blalock, Jr. (Ed.), Causal models in the social sciences. Chicago: Aldine, 1971.

Alwin, D. F. Approaches to the interpretation of relationships in the multitrait-multimethod matrix. In H. L. Costner (Ed.), Sociological Methodology 1973-1974. San Francisco: Jossey-Bass, 1974.

Campbell, D. T., \& Fiske, D. W. Convergent and discriminant validation by the multitrait-multimethod matrix. Psychological Bulletin. 1959, 56. 81-105.

Jöreskog, K. G. A general approach to confirmatory maximum likelihood factor analysis. Psychometrika, 1969, 34, 183-202.

Jöreskog, K. G. A general method for analysis of covariance structures. Biometrika, 1970,57, 239-251.

Jöreskog, K. G. A general method estimating a linear structural equation system. In A. S. Gold berger \& O. D. Duncan (Eds.), Structural equation models in the social sciences. New York: Seminar Press, 1973.

Jöreskog, K. G., \& VanThillo, M. LISREL-A general computer program for estimating a linear structural equation system involving multiple indicators of unmeasured variables. (Research Bul- 
letin 72-56). Princeton. NJ: Educational Testing Service, 1972.

Jöreskog, K. G., \& Goldberger, A. S. Estimation of a model with multiple indicators and multiple causes of a single latent variable. Journal of the American Statistical Association. 1975, 70. 631-639.

Kalleberg, A. L., \& Kluegel, J. R. Analysis of the multitrait-multimethod matrix: Some limitations and an alternative. Journal of A pplied Psychology. 1975, 60, 1-9.

Kenny, D. A. An empirical application of confirmatory factor analysis to the multitrait-multimethod matrix. Journal of Experimental Social Psychology, 1976, 12, 247-252.

Kothandapani, V. Validation of feeling, belief, and intention to act as three components of attitude and their contribution to the prediction of contraceptive behavior. Journal of Personality and Social Psychology, 1971, 19, 321-333.

Lawler, E. E. The multitrait-multirater approach to measuring managerial job performance. Journal of Applied Psychology. 1967, 51. 369-381.
Ostrom, T. M. Relationship between affect, behavior, and cognition. Iournal of Experimental Social Psychology, 1969, 5, 12-30.

Schmidt, F. L., \& Johnson, R. H. Effect of race on peer ratings in an industrial situation. Journal of Applied Psychology, 1973, 57, 237-241.

Tucker, L. R., \& Lewis, C. A reliability coefficient for maximum likelihood factor analysis. Psychometrika, 1973, 38, 1-10.

Werts, C. E., \& Linn, R. L. Path analysis: Psychological examples. Psychological Bulletin, 1970, 74. 193-212.

Werts, C. E., Jöreskog, K. G., \& Linn, R. L. Analyzing ratings with correlated intrajudge measurement errors. Educational and Psychological Measurement, 1976, 36, 319-328.

\section{Author's Address}

Neal Schmitt, Department of Psychology, Olds Hall, Michigan State University, East Lansing, MI 48824 